\title{
A Discreet Wearable Long-Range Emergency System Based on Embedded Machine Learning
}

Orfanidis, Charalampos; Hassen, Rayen Bel Haj ; Kwiek, Armando; Fafoutis, Xenofon; Jacobsson, Martin

\section{Published in:}

Proceedings of the $5<$ sup $>$ th $</$ sup $>$ IEEE PerCom Workshop on Pervasive Health Technologies

Publication date:

2021

Document Version

Peer reviewed version

Link back to DTU Orbit

Citation (APA):

Orfanidis, C., Hassen, R. B. H., Kwiek, A., Fafoutis, X., \& Jacobsson, M. (2021). A Discreet Whearable LongRange Emergency System Based on Embedded Machine Learning. In Proceedings of the 5 IEEE PerCom Workshop on Pervasive Health Technologies IEEE.

\section{General rights}

Copyright and moral rights for the publications made accessible in the public portal are retained by the authors and/or other copyright owners and it is a condition of accessing publications that users recognise and abide by the legal requirements associated with these rights.

- Users may download and print one copy of any publication from the public portal for the purpose of private study or research.

- You may not further distribute the material or use it for any profit-making activity or commercial gain

- You may freely distribute the URL identifying the publication in the public portal 


\title{
A Discreet Wearable Long-Range Emergency System Based on Embedded Machine Learning
}

\author{
Charalampos Orfanidis ${ }^{\dagger}$, Rayén Bel Haj Hassen ${ }^{\ddagger}$, Armando Kwiek ${ }^{\ddagger}$, \\ Xenofon Fafoutis ${ }^{\dagger}$, Martin Jacobsson ${ }^{\ddagger}$ \\ $\dagger$ Department of Applied Mathematics and Computer Science, Technical University of Denmark \\ $\ddagger$ School of Engineering Sciences in Chemistry, Biotechnology and Health, KTH Royal Institute of Technology
}

\begin{abstract}
Low-Power Wide Area Networks have contributed in several parts of the Internet of Things ecosystem during the last years by enabling long range, robust and low power communication. Machine Learning for embedded systems has also assisted the advancement of the Internet of Things by identifying patterns and increasing the accuracy of predicting events and behaviours. At the same time, wearable and mobile systems are less obtrusive, consuming less energy and have more computing resources. In this paper we combine these three components and propose a low cost wearable system based on a regular shoe and off-the-shelf electronics which is able to recognize foot gestures and transmit messages over long range, in cases of emergency. The evaluation considers an application scenario where the user performs specific foot gestures to trigger the transmission of an emergency message, during other activities (e.g., walking). The proposed wearable system would benefit a user who is in danger and attempts to notify her/his emergency contacts in a discreet manner. Results show that the proposed system is able to identify the intended foot gestures with $98 \%$ accuracy.
\end{abstract}

Index Terms-IoT, LPWAN, Pervasive Health, Wearable, Foot Gesture

\section{INTRODUCTION}

Low-Power Wide Area Networks (LPWANs) have enabled battery powered long range communication for the Internet of Things (IoT) [22]. The lifetime of a node, which is powered solely by a battery, may be from 2 to 4 years approximately [20], depending on the selected configuration. Furthermore, LPWANs can operate in urban environments where the network density and interference level might be high [8], [23]. Smart city applications like distributing energy depending on people demands [5], smart parking models which predict available free positions based on previous data and many more have emerged to improve daily life and help on the current climate change crisis [17]. LPWANs are also being used in application scenarios involving health and activity monitoring [14]. The advantages of using LPWAN in health and activity monitoring are that they offer high robustness and reliability and the coverage is significantly larger [24] compared to Bluetooth Low Energy (BLE) or IEEE 802.15.4, which typically are used in similar situations. Due to the long range communication, we can assume that in an smart city environment, where gateways are abundant, LPWANs systems which are designed to monitor health or activity, do not depend on any short range gateway or smartphone devices. Thus, a wearable system including an LPWAN can operate more "independently" in such cases.

Machine Learning (ML) for embedded systems and IoT have lately emerged and today there are lightweight versions of ML models [18]. Hardware accelerators are designed [15] and software libraries [2] are developed specifically to support ML for IoT applications. TinyML [32] is a foundation that is designed to support ultra-low power ML applications for embedded systems. Another reason for moving the ML models and computation from the cloud to the end devices is that 5 quintillion bytes of data are produced every day from IoT devices and less than $1 \%$ of unstructured data is analyzed or used at all [6], [10]. Hence, moving the ML model to the end device and the sensors, where these data are generated will make it easier to utilize them. Another interesting paradigm is to move a part of the computation to the network edge devices in order to increase the resources and execute more complex ML models [33]. However, this approach is introducing new tradeoffs due to the communication with the other devices and this tradeoff hinders applications with hard deadlines like real-time systems or the emergency system that this paper focus on.

In this paper, we present a wearable system including a ML-based foot gesture interface combined with a LoRa radio. LoRa [4], [28] is a LPWAN technology widely used in IoT as it offers long range and robust communication in low-power. The ML model is designed to execute solely on embedded Microcontroller Unit (MCU) resources. The proposed system might be utilized in a use case scenario where the user is in danger and wants to broadcast a message asking for help. However, the user might not want to be noticed by a possible perpetrator. Furthermore, since LPWAN has long range coverage (in smart cities environments), the user does not need to carry a smartphone or being depended on any short range gateway, which is the case for many wearables designed for outdoor sports/activities. 
A key challenge in existing gesture recognition applications is to distinguish the pattern of the desired gesture and not misinterpret it with similar patterns caused by other activities, like jogging for instance. To avoid this, we propose a classifier based on a lightweight Neural Network (NN), which is able to distinguish between 3 activities (walking, jogging, standing) and 2 gestures (double tap at toe tip and double tap at heel) in different environments. The illustrated proof of concept outlines that this approach can be used in other use case scenarios.

Our contributions, which we believe are beneficial for pervasive health and activity monitoring applications, are listed below:

- An unobtrusive, low cost prototype that can operate independently (in smart city environments) which is able to capture and classify several activities and foot gestures with high accuracy.

- A classifier based on a lightweight NN model which is implemented and tuned in Python first and then ported to the MCU of the prototype with $98 \%$ accuracy on classifying activities from foot gestures.

- A preliminary in-the-wild evaluation of the classifier conducted indoors and outdoors by two individuals.

\section{RELATED WORK}

The related works can be divided in two areas. First, emergency systems designed for the same or similar purpose are presented and compared with the proposed system. Second, ML for embedded systems which are designed for several purposes are presented and we focus on how a ML model can be merged in embedded systems and what the potential benefits are.

Silent Beacon [29] is a personal alert system that consists of a button designed to communicate through Bluetooth with a smartphone. In case of emergency it can contact a number of contacts and share the current GPS position. Run angel is a similar product, but focuses on outdoors sports users [27]. The wearable is a wristband and beside sending emergency messages and the position of the user in case of danger, it also emits a very loud alarm to attract attention. In 2016, Harvard medical school classified available alert systems (mostly for elderly) based on their cost [11]. The wearables were either designed for neck or wristbands and the main difference between them is the communication range and the fact that some of them provide fall detection. The scientific literature focuses mostly on the elderly and proposes similar approaches to fall detection. For instance, Ren et al. [25] designed a wearable, which is attached to the belt and based on accelerometers and the implemented algorithm is able to detect fall with an accuracy of $96 \%$. An approach, which is closer to the one we propose, is presented in [31], where they propose a wearable based on LPWAN to monitor individuals who do outdoor activities in remote and risky environments, such as hiking, skiing, etc. In this system, there is a synergy between a smartwatch monitoring health metrics, an LPWAN for sharing GPS signals, and a smartphone for visualizing possible alerts.

Several of the solutions mentioned above depend on a monthly fee, which include maintenance costs and sometimes a GSM subscription. Moreover, most of the systems are implemented based on indiscreet wearables or the communication range is rather short based on a smartphone or a gateway with short range. This means that in case of emergency, the user might be observed by a possible perpetrator. Furthermore, if the system depends on a smartphone and the user forgot it during the activity, the alert system does not work. In contrast, the long range emergency system that we propose can operate independently in smart city environments at a lower cost and the interface is rather discreet.

In [18], the authors presents the progress that have been made to overcome a number of challenges with deep learning when used on embedded and mobile devices. The authors mention challenges that the community faced in the beginning working with smartphones, then continued with smartwatches and concluding with what were the trends at the time. Binarized Neural Networks are performing feedfoward inference, they can occupy a large amount of memory, but in [21], the authors classify sensor data directly on the end device instead of transmitting the sensor data to the cloud. In order to minimise the memory usage, they use 1-bit weights instead of 32-bit floating points weights. Furthermore, by changing the order of inference computation, they decreased the size of the temporaries, which store the computations between layers during feedforward inference, while still achieving 95\% accuracy.

ML is used in wearable systems to capture events of interests in physical activities or medical complications. Rockni et al. [26] tackle the problem of retraining wearable systems where a new sensor is introduced to the network and is deployed in several parts of the body. They introduce a method to transfer activity recognition data from the sensors which are already deployed to the new one. The authors of [9] attempt to decrease the computational resources required in multi-task learning approaches. They focus on audio tasks (i.e., speech recognition and emotion recognition) and how they can share representations among tasks while having a balance in performance. An on-board activity classification framework for wearable systems is designed to decrease the energy consumption [34]. The energy consumption is decreased by performing the classification on board and consequently by transmitting less data through the radio. Moreover, they enable a conditional classification where energy-hungry components will be activated only 
if it would be beneficial, based on a classification which takes place previously and requires less energy. Suresh et al. in [30] illustrate that an on-board classification in smart farming applications can extend the battery lifetime by a factor of three. In a similar spirit with the previous approaches, transmitting less data through the radio decreases the energy consumption.

The novelty of the our system does not lie in a new ML compression technique that reduces the memory footprint or the consumed energy but in the combination between a lightweight ML model, which is able to be deployed on-board into an embedded system, a foot gesture interface and an LPWAN, which is able to operate robustly and reliably as an emergency system. All the aforementioned approaches act as evidence that embedded ML has several benefits for the IoT ecosystem.

\section{PRoblem Formulation}

Using a wearable system to recognize foot gestures that is based on low cost force sensors placed underneath a shoe sole may be challenging. The wearable system is designed for use cases, such as jogging, walking, or other sports/activities in different environments. In order to distinguish the input from the force sensors between these activities and the desired foot gestures, we decided to use a NN classifier. Implementing a NN model on a resource-constrained IoT node is a non-trivial process because it is not designed to function under limitations imposed by an MCU in terms of memory, computing power and energy resources.

The research question we are addressing in this paper is if a user may utilize a long range emergency system like the one briefly described above, under use cases in sports, pervasive health or a similar scenarios, despite the resource-constrained embedded system. Furthermore, we evaluate the developed system under these scenarios to quantify the accuracy in distinguishing between foot gestures and other activities.

\section{System OVERVIEW}

This section describes the technical details of the system. During the design, we selected low cost and low power consumer electronics in order to fit into the IoT ecosystem. The system can be divided into two parts, the hardware prototype and the NN classifier.

\section{A. Hardware prototype}

To realize the prototype, we use a regular shoe and then we place two force sensors underneath the shoe sole. One is placed at the toe tip and the other at the heel as depicted in Figure 1. The force sensors are connected to the LPWAN MCU, which is glued to the side of the shoe with a small power-bank. The model of the force sensors is FSR 406 from Interlink Electronics and have a surface of $38 \mathrm{~mm} \times 38 \mathrm{~mm}$ in square shape.
The LPWAN device consists of an ESP32 MCU and a RFM95 LoRa modem. Note that another LPWAN is used as a receiver which is connected directly to a laptop to act as a gateway. The software we use is Arduino-LMIC library [1].

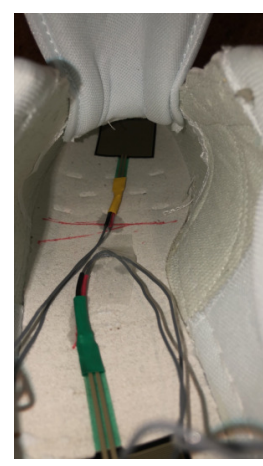

(a)

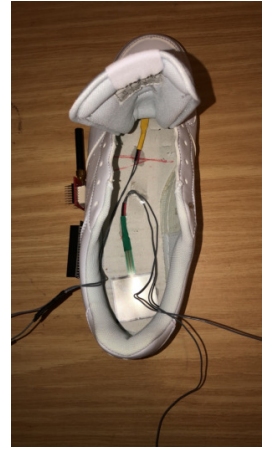

(b)

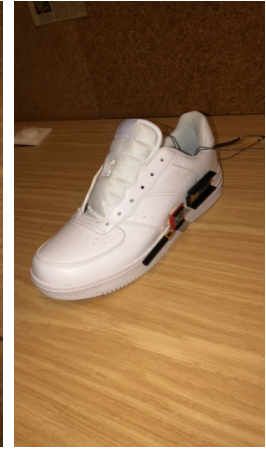

(c)
Fig. 1: The prototype was based on a regular shoe including two force sensors, one at the the tip of the shoe (a), one at the heel (b), and both are connected on an IoT node (c)

\section{B. Neural Network classifier}

The use of Neural Networks on mobile and embedded devices have increased a lot during the last years [19]. This integration has lead to scientific breakthroughs and has shaped the norm in pattern recognition and other features offered from NNs in IoT and pervasive health. In our approach, we use a $\mathrm{NN}$ classifier with two hidden layers that operates by forwarding information in one direction through each layer in the network. This model was chosen among others ML approaches (Long-Short Term Memory [12], Convolutional Neural Network [3], and even Support Vector Machine [7]) by evaluating the tradeoff between accuracy and implementation complexity to fit on an ESP32 MCU. The approach we followed here was to first implement the model in Python and the Keras [16] library in order to evaluate and configure it before porting it to the MCU.

In order to define the NN classifier, the first step was to acquire a dataset and use it to train a $\mathrm{NN}$ model. Using the prototype, we performed the activities and the desired gestures. The analog output voltage from the sensors were converted to digital values via the Analog to Digital Converter (ADC), which provided a digital value between 0 - 4095. We collected the data at a rate of $50 \mathrm{~Hz}$ and went on for approximately 10 minutes, leading to 30,000 stored data points. Data was then split into batches of 100 sample points ( 2 seconds of recording) creating a total of 300 arrays for each activity/gesture. This was done for both force sensors. Furthermore, the data was gathered in a controlled environment using a treadmill indoors, but also outdoors 


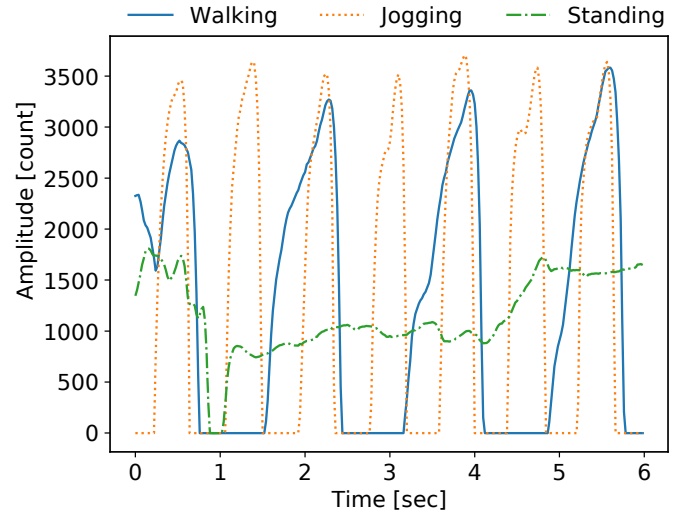

(a) Front sensor

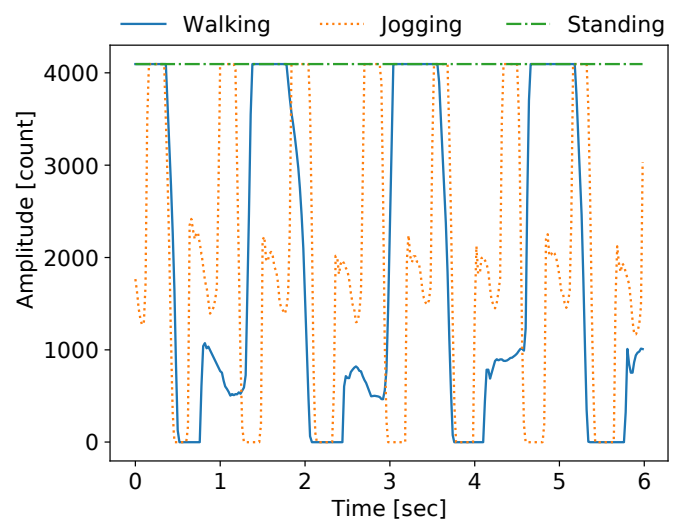

(b) Back sensor

Fig. 2: Raw measurements of the investigated activities from both sensors

using a street pavement in a regular urban environment. The activities performed were walking, jogging, standing and the gestures were double tap at the tip of the sole and double tap at the heel. The time interval between the taps was approximately 0.5 seconds. Moreover, a label was given manually to each activity/gesture and the dataset was generated from two individuals performing the mentioned activities and gestures. A part of the collected data are illustrated in Figure 2 and Figure 3. In Figure $2 b$, we see that the force sensor saturates when the user was standing. This did not affect the accuracy of our results. However, we have to mention that if we target more complex gestures, the accuracy might drop. This can be solved by using a sensor which is able to tolerate more weight.

The Figures 2-3 illustrate that it is difficult to distinguish foot gestures between activities based on the raw data. For instance, the double tap heel signal coming from the back sensor in Figure $3 b$ could be easily misinterpreted for jogging in Figure $2 b$ if the jogging was faster. To solve this, we extracted a number of features to increase the accuracy of the model: median,

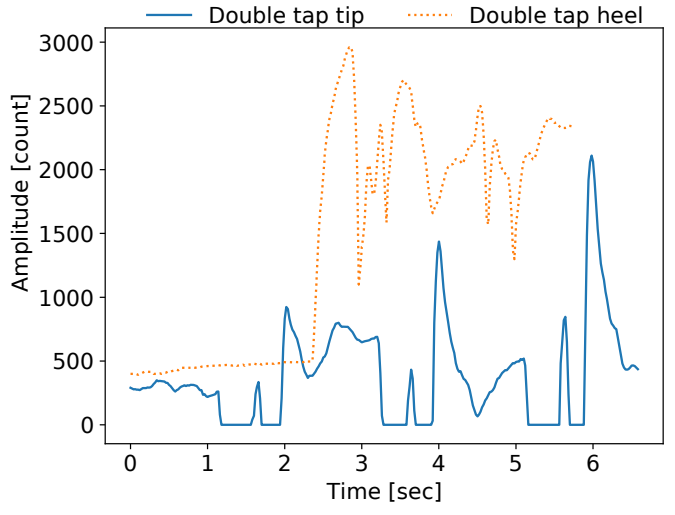

(a) Front sensor

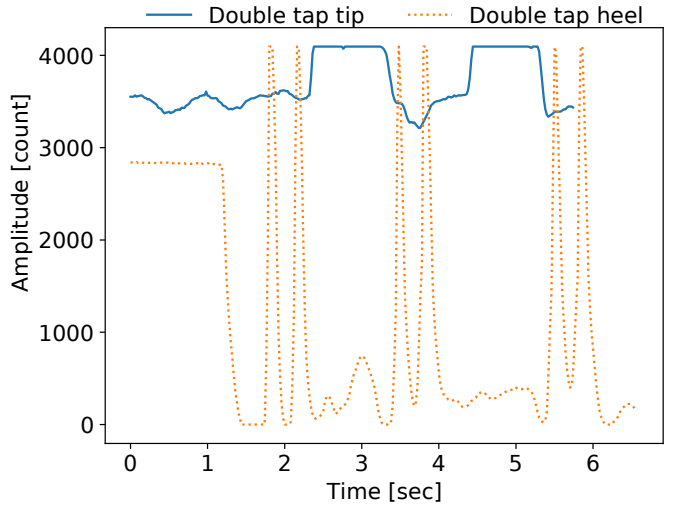

(b) Back Sensor

Fig. 3: Raw measurements from the sensors during performing foot gestures: double tap at the tip and the heel captured by the (a) front and (b) back sensor

mean, standard deviation, max, min, and number of steps. After consulting a correlation matrix, we dropped the following features: the max and min from the front sensor and the min from the back sensor. This was a step to make the model more lightweight in order to port it to the MCU and also because these features did not contribute to the classification process. Thus, the model has 4 layers including two hidden ones. The input layer has 9 nodes which is the number of the features. The first hidden layer has 32 nodes and the second has 16 nodes. Finally, the output layer has 5 nodes, which is the number of activities/gestures. As an activation function, we used rectified linear unit as it showed to have the minimum loss and cross-entropy loss function for the same reason. We also used ridge regression to avoid overfitting.

\section{Pilot Evaluation}

During the evaluation, we followed one experimental scenario indoors and one outdoors, executed by two individuals. The scenario was the following: An individual is using the shoe and tried to perform each activity/gesture 


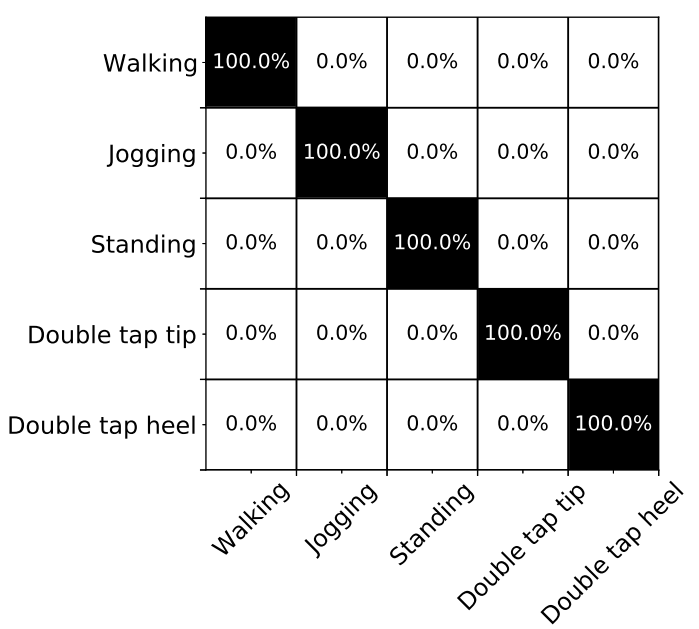

(a) Person A

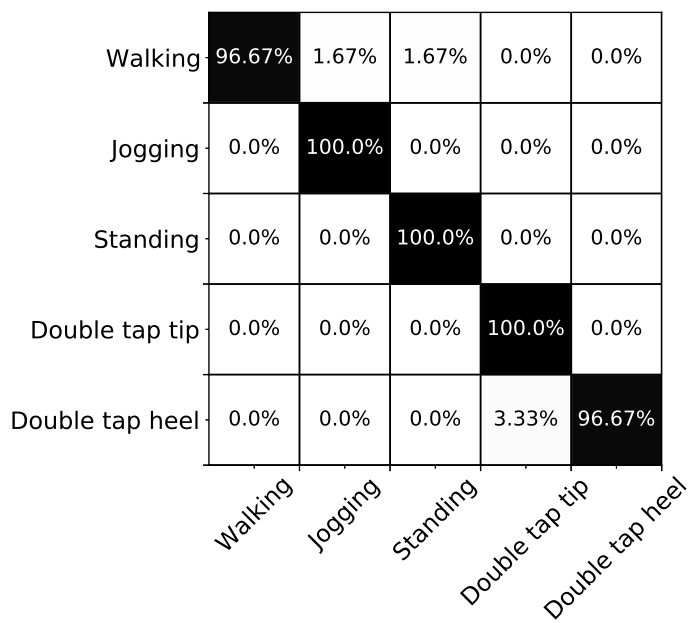

(b) Person B

Fig. 4: Experimental results from indoors, on the Yaxis is the ground truth and on the $\mathrm{X}$-axis, the predicted output

60 times. Performing an activity 60 times is equal to 2 minutes of walking, standing, or jogging. The foot gestures were performed every 2 seconds approximately and with 0.5 second interval between the taps. Even though we managed to port the $\mathrm{NN}$ classifier to the MCU, we choose to take raw values during the experiments and do the classification offline in order to perform a more systematic analysis on the statistics of the results. We used $80 \%$ for training the NN classifier and $20 \%$ for testing. Note that the classifier is trained per individual. We followed this approach because gait pattern is a unique biometric [13] and creating a universal classifier was not the scope of this paper, but rather proposing a lightweight NN model that performs well with the wearable system and the given scenarios. Furthermore, it is common in foot gesture interfaces that the users are programming the specific gestures they want and there

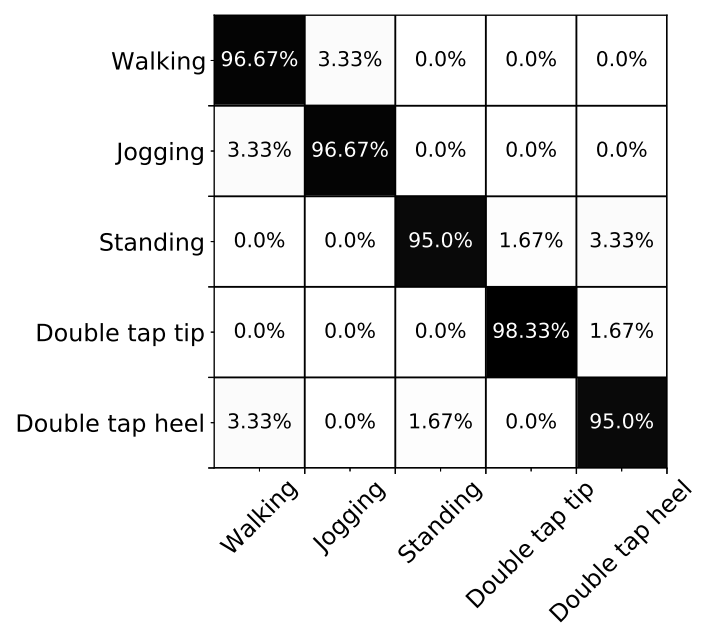

(a) Person $\mathrm{A}$

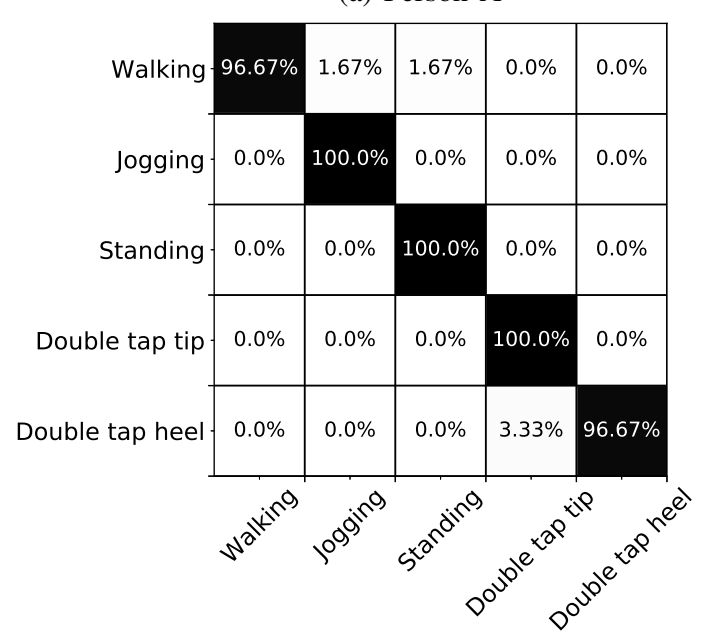

(b) Person B

Fig. 5: Experimental results from outdoors, on the Yaxis is the ground truth and on the $\mathrm{X}$-axis, the predicted output

is an offered a procedure to register it. We repeated this scenario indoors and outdoors and we plot the confusion matrices of the results in Figures 4 - 5 .

Figure 4 presents the accuracy of the two individuals indoors and we see that all the activities/gestures are classified with more than $96.67 \%$ accuracy and the overall accuracy for both is $99.33 \%$. We have to mention that for the indoor cases, the environment was more controlled as we used a treadmill for the experiments. For the outdoors case in Figure 5, there is a slight drop of the overall accuracy to $97.5 \%$ and also in this case all the activities/gestures are classified with more than 95\% accuracy. Even though the outdoor environment is more unstable, the accuracy drop is almost insignificant and illustrates that the interface can function in several environments. However in a critical scenario like this, the accuracy should be higher and we plan to investigate 
how to improve it.

\section{CONCLUSION}

In this paper, we presented a long range emergency system that consists of a regular shoe wearable, a foot gesture interface, and an LPWAN. The whole implementation is based on off-the-shelf and low cost electronics. The results show that with a lightweight NN classifier, it is possible to offer approximately $98 \%$ accuracy among 5 different activities/gestures tested by two individuals indoors and outdoors. Based on the obtained results we can say that the proposed wearable system is able to to deliver robust and reliable performance as a long range emergency system. In addition, in a smart city environment it can operate independent from a smartphone or a short range gateway which makes it more practical towards users in outdoor sports or activities. Moreover, we plan to evaluate the proposed system further with more individuals and more use case scenarios to get more insights.

\section{REFERENCES}

[1] Arduino-LMIC library. https://github.com/matthijskooijman/ arduino-lmic, 2020. Accessed: 2021-29-01

[2] Arm. ARM COMPUTE LIBRARY, 2019. https://www.arm.com/ why-arm/technologies/compute-library.

[3] C. M. Bishop. Pattern Recognition and Machine Learning (Information Science and Statistics). Springer-Verlag, Berlin, Heidelberg, 2006.

[4] M. Bor, J. Vidler, and U. Roedig. LoRa for the Internet of Things. In Proceedings of the 2016 International Conference on Embedded Wireless Systems and Networks, EWSN '16, page 361-366, USA, 2016. Junction Publishing.

[5] G. D. Campo, I. Gomez, S. Calatrava, R. Martinez, and A. Santamaria. Power Distribution Monitoring Using LoRa: Coverage Analysis in Suburban Areas. In Proceedings of the 2018 International Conference on Embedded Wireless Systems and Networks, EWSN '18, page 233-238. Junction Publishing, 2018.

[6] Cisco. Internet of Things (IoT) Data Continues to Explode Exponentially. Who Is Using That Data and How?, 2018. https://news-blogs.cisco.com/datacenter/internet-of-things-iotdata-continues-to-explode-exponentially-who-is-using-thatdata-and-how.

[7] C. Cortes and V. Vapnik. Support-vector networks. Machine Learning, 20(3):273-297, Sep 1995.

[8] R. Eletreby, D. Zhang, S. Kumar, and O. Yağan. Empowering Low-Power Wide Area Networks in Urban Settings. In Proceedings of the Conference of the ACM Special Interest Group on Data Communication, SIGCOMM '17, page 309-321, New York, NY, USA, 2017. Association for Computing Machinery.

[9] P. Georgiev, S. Bhattacharya, N. D. Lane, and C. Mascolo. Low-resource multi-task audio sensing for mobile and embedded devices via shared deep neural network representations. Proc. ACM Interact. Mob. Wearable Ubiquitous Technol., 1(3), Sept. 2017.

[10] Harvard Business Review. What's Your Data Strategy?, 2017. https://hbr.org/2017/05/whats-your-data-strategy.

[11] Harvard Health Publishing. The new generation of wearable medical alert systems, $2016 . \quad$ https: //www.health.harvard.edu/healthy-aging/the-new-generationof-wearable-medical-alert-systems.

[12] S. Hochreiter and J. Schmidhuber. Long Short-Term Memory. Neural Computation, 9(8):1735-1780, 1997.

[13] F. Horst, S. Lapuschkin, W. Samek, K.-R. Müller, and W. I. Schöllhorn. Explaining the unique nature of individual gait patterns with deep learning. Scientific Reports, 9(1):2391, Feb 2019.
[14] T. Hossain, M. A. R. Ahad, T. Tazin, and S. Inoue. Activity Recognition by Using LoRaWAN Sensor. In Proceedings of the 2018 ACM International Joint Conference and 2018 International Symposium on Pervasive and Ubiquitous Computing and Wearable Computers, UbiComp '18, page 58-61, New York, NY, USA, 2018. Association for Computing Machinery.

[15] Intel. Intel® Movidius Vision Processing Units (VPUs), 2016. https://www.intel.com/content/www/us/en/products/processors/ movidius-vpu.html.

[16] Keras. Keras: The python deep learning library, 2020.

[17] Københavns kommune. Smart City, 2017. https:// urbandevelopmentcph.kk.dk/indhold/smart-city.

[18] N. D. Lane, S. Bhattacharya, A. Mathur, P. Georgiev, C. Forlivesi, and F. Kawsar. Squeezing Deep Learning into Mobile and Embedded Devices. IEEE Pervasive Computing, 16(3):82-88, 2017.

[19] N. D. Lane and P. Warden. The Deep (Learning) Transformation of Mobile and Embedded Computing, year $=2018$, volume $=51$, number $=5$. Computer, pages 12-16.

[20] J. C. Liando, A. Gamage, A. W. Tengourtius, and M. Li. Known and Unknown Facts of LoRa: Experiences from a Large-Scale Measurement Study. ACM Trans. Sen. Netw., 15(2), Feb. 2019.

[21] B. McDanel, S. Teerapittayanon, and H. Kung. Embedded Binarized Neural Networks. In Proceedings of the 2017 International Conference on Embedded Wireless Systems and Networks, EWSN '17, page 168-173, USA, 2017. Junction Publishing.

[22] K. Mekki, E. Bajic, F. Chaxel, and F. Meyer. A comparative study of LPWAN technologies for large-scale IoT deployment. ICT Express, 5(1):1 - 7, 2019.

[23] C. Orfanidis, L. M. Feeney, M. Jacobsson, and P. Gunningberg. Investigating interference between LoRa and IEEE 802.15.4g networks. In 2017 IEEE 13th International Conference on Wireless and Mobile Computing, Networking and Communications (WiMob), pages 1-8, 2017.

[24] J. Petäjäjärvi, K. Mikhaylov, M. Hämäläinen, and J. Iinatti. Evaluation of LoRa LPWAN technology for remote health and wellbeing monitoring. In 2016 10th International Symposium on Medical Information and Communication Technology (ISMICT), pages 1-5, 2016.

[25] L. Ren, Q. Zhang, and W. Shi. Low-Power Fall Detection in Home-Based Environments. In Proceedings of the 2nd ACM International Workshop on Pervasive Wireless Healthcare, MobileHealth '12, page 39-44, New York, NY, USA, 2012. Association for Computing Machinery.

[26] S. A. Rokni and H. Ghasemzadeh. Synchronous Dynamic View Learning: A Framework for Autonomous Training of Activity Recognition Models Using Wearable Sensors. In Proceedings of the 16th ACM/IEEE International Conference on Information Processing in Sensor Networks, IPSN '17, page 79-90, New York, NY, USA, 2017. Association for Computing Machinery.

[27] runangel. runangel, 2018. https://runangel.com/.

[28] Semtech. LoRaWAN. https://lora-alliance.org/resource-hub/ lorawantm-specification-v11, 2020. Accessed: 2020-04-17.

[29] Silent Beacon. Introducing the Silent Beacon Panic Button, 2019. https://silentbeacon.com/.

[30] V. M. Suresh, R. Sidhu, P. Karkare, A. Patil, Z. Lei, and A. Basu. Powering the IoT through embedded machine learning and LoRa. In 2018 IEEE 4th World Forum on Internet of Things (WF-IoT), pages 349-354, 2018.

[31] G. B. Tayeh, J. Azar, A. Makhoul, C. Guyeux, and J. Demerjian. A wearable lora-based emergency system for remote safety monitoring. In 2020 International Wireless Communications and Mobile Computing (IWCMC), pages 120-125, 2020.

[32] tinyML Foundation. Enabling ultra-low Power Machine Learning at the Edge, 2019. https://www.tinyml.org/home/index.html.

[33] X. Wang, Y. Han, V. C. M. Leung, D. Niyato, X. Yan, and $\mathrm{X}$. Chen. Convergence of Edge Computing and Deep Learning: A Comprehensive Survey. IEEE Communications Surveys Tutorials, 22(2):869-904, 2020.

[34] P. Zalewski, L. Marchegiani, A. Elsts, R. Piechocki, I. Craddock, and X. Fafoutis. From Bits of Data to Bits of Knowledge-An On-Board Classification Framework for Wearable Sensing Systems. Sensors, 20(6):1655, Mar 2020 